\title{
Optimal Placement of Phasor Measurement Unit for Observation Reliability Enhancement
}

\author{
Van-Khoi TRAN*, He-sheng ZHANG ${ }^{\dagger}$ and Van-Nghia NGUYEN**
}

\begin{abstract}
Phasor Measurement Unit (PMU) placement is a crucial problem for State Estimation (SE) of the power system, which can ensure that the power network is fully observed. Further, the observation reliability problem of the system has been concerned in the operation conditions. In this paper, based on modified weighted adjacent matrix $\left(\mathrm{A}_{\mathrm{w}}\right)$, an optimal placement method is proposed to solve simultaneously two problems involving the optimal PMU placement problem and the observation reliability enhancement problem of the system. The purpose of the proposed method is to achieve both the minimum total cost and the maximum observation reliability, with a focus on increasing the security of observability, strengthening the observation reliability of buses as well as enhancing the effectiveness of redundancy. Simulations on IEEE 14, 24, 30 and 57 bus test systems are presented to justify the methodology. The results of this study show that the proposed method is not only ensuring the power network having the observability effectively but also enhancing significantly the observation reliability. Therefore, it can be a useful tool for SE of the power system.
\end{abstract}

Keywords: Phasor measurement unit (PMU), Optimal placement, Local search algorithm (LSA), Modified weighted adjacent matrix, Observation reliability

\section{Introduction}

Optimal placement of Phasor Measurement Unit (PMU) has been invested and applied in the field of the Power System Observability (PSO) for a recent decade. The common goal of placement method is to achieve the PSO by using the minimal number of PMUs in order the minimum cost. Integer linear programming is one of the dominant mathematical optimization technique, which is commonly used for solving effectively [1-5]. Heuristic and meta-heuristic search algorithms also have been attracted a lot of concerns in solving the PMU placement problem due to their good characteristics [6-9]. Relate to robust and efficient State Estimation (SE), many requirements should be regarded in addition to the number of PMUs. On the other hand, in considering the system reliability, it can be noted that the probabilities of PMU failure, communication channels, and line outage need be interested. In this case, the measurements set, which is calculated according to a single PMU, can be unreliable. For this reason, the bus observation reliability should be considered in the PMU placement problem.

With the aim of finding out the PMU placement which achieves the best observable reliability, the authors in $[4$, 10-12] proposed solutions to get the maximum redundancy

$\dagger$ Corresponding Author: School of Electrical Engineering, Beijing Jiaotong University, China. (hszhang@bjtu.edu.cn)

* School of Electrical Engineering, Beijing Jiaotong University, China. (15119005@bjtu.edu.cn)

** Dept. of Electrical Engineering, University of Transport and Communications, Vietnam. (nguyennghia.ktd@utc.edu.vn)

Received: July 7, 2016; Accepted: January 17, 2017 in the network observability. These studies could find the best redundant case; however, the redundancy is passive because it happens casually when solving the observability problem.

An optimal placement method which is based on the branch was proposed by Gholiha [13]. Although this method can achieve the maximum security of the observability in the contingency mode, its drawback is, one PMU only observes two buses so it has not measurement redundancy, and the total cost is increased by using a large number of PMUs.

Recently, the authors in reference [14] aimed to enhance the observation reliability of the system by considering the redundancy is an extra objective function in the optimization problem. This solution is based on the required number of redundancies to find out the minimum number and the placement of PMUs. However, as same as the solution in [4, 10-12], the redundancy is still passive; it cannot implement to obtain the desired redundancy for the specific buses.

This work was extended and developed with the solution of measurement redundancy for all system, in which case that considers the random failure of PMUs or line outage $[12,15-17]$. This solution strengthens significantly the bus observation reliability; however, it does not need to implement enough redundancy for all buses to ensure the system observation reliability because the reliability of each line and the importance of each bus are not the same.

In all previous studies, the buses or lines in a power network are on the same property level; the bus observation reliability only depends on the reliability of PMU. Xu et al. 
[18] pointed out that there are some buses and lines in the power network that have an important impact on the reliability of system because they reflect the characteristic of the power system. The state of the system can be assessed from the information of these elements. That means the characteristics of power network also have certain impacts on the system observation reliability. It is not much significance when a bus with low importance having measurement redundancy, or one PMU channel installed at two lines with two different reliabilities that its observation reliability is certainly not similar; therefore, beside the redundant solution, a solution which finds out the important buses and the high reliable lines to install PMU, can enhance the observation reliability for buses, too.

Based on the modified weighted adjacent matrix that contains the characteristics of the power network, this paper proposes a new method to solve the optimal PMU placement problem. The proposed method optimizes both the number of PMUs and number of PMU branches. The purpose of the proposed solution is to achieve the minimum total cost and the maximum observation reliability, with a focus on increasing the security of observability, strengthening the observation reliability of buses as well as enhancing the effectiveness of redundancy. The process of algorithm consists of three stages: The first stage forms the weighted adjacent matrix by determining the importance of buses and the reliability of branches in power network. The second stage is based on the reliability of branches and the importance of buses to optimize the number of PMUs and their placement in electric power system with ensuring full network observability and redundant requirements. The third one optimizes the branches of PMUs to achieve minimum total cost.

This paper improves the method from the passive redundancy to the active redundancy for buses; besides that, it uses the Prim algorithm to specify the most reliable channel for each bus. The combination of the active redundancy and the most reliable channel can maximize the observation reliability for buses and heighten the observation reliability of a power system to achieve the desired observation reliability; therefore, the proposed method is not only ensuring the effective observability but also maximizing the observation reliability of the system.

The remainder of this paper begins with an introduction of the theoretical approach in Section 2 and then presents the proposed algorithm in Section 3. Subsequently, the simulation results are shown in Section 4 and discussed in Section 5. Finally, the conclusions are drawn in Section 6.

\section{Theoretical Approach}

\subsection{Modified weighted adjacent matrix}

A power grid can be represented by a graph, which consists of a set of nodes (buses) and a set of edges (branches). In this model, the set of edges describes the transmission lines connecting between the buses of the power system. For simplifying in programming, a matrix is used to describe the bus connectivity information of power system, it can be named as an adjacent matrix (A).

Here, we form a modified weighted adjacency matrix $\left(A_{w}\right)$ for the purpose of which optimizing the placement of PMUs in power grid network as follow:

$$
A_{w}=\left[\begin{array}{cccccc}
A_{11} & A_{12} & A_{13} & \ldots & \ldots & A_{1 n} \\
A_{21} & A_{22} & A_{23} & & & A_{2 n} \\
A_{31} & A_{32} & A_{33} & & & A_{3 n} \\
\ldots & \ldots & \ldots & \ldots & & \\
A_{n 1} & A_{n 2} & A_{n 3} & & & A_{n n}
\end{array}\right]
$$

where $A_{w}$ is a square matrix of order $n$ which expresses the importance of buses and the reliability of branches in network; $n$ is number of buses in power network; $A_{k k}$ is the importance of bus $K(k=1, \ldots, n)$ and $A_{k l}(k \neq 1)$ is the reliability of line connected between bus $K$ and bus L ( $A_{k}=0$ when bus $K$ and bus $L$ are not connected).

Important buses are defined as they have an important impact on the characteristic and the reliability of power grid. Similar to [19-22], the centrality measure technique is also used to obtain the relative importance of nodes in the network. In this study, we improve the formula to calculate the importance of bus in power system through an index called Observation Degree Index (ODI). ODI of bus $v$ is given by:

$$
O D I(v)=\frac{\sum_{s \neq t \neq v} P_{s t}(v) / \sigma_{s t}}{P_{\sum \text { load }}}
$$

where $\sigma_{s t}$ is the number of shortest paths from generation bus " $s$ " to load bus " $t$ ", $P_{s t}(v)$ is the amount of power from the mentioned paths that conducts pass through bus $v$; and $P_{\text {Eload }}$ is a total of load power in the system.

$O D I(v)$ is known as the importance of bus $v$ in power grid. When the value of $O D I(v)$ is higher, it means that bus $\mathrm{V}$ is more important.

The reliability $(\rho)$ of one line is generally calculated according to its characteristics (power and length) as follows:

$$
\rho=e^{-\lambda \cdot T}
$$

where $\lambda$ is outage rate of the line in a time duration $T$ ( $T$ is normally selected as one year)

Rely on the result of reference [18] and the idea of reference [23] the reliability of line is improved as below:

$$
\rho=e^{-\left(\lambda+C_{b}(e)\right) \cdot T}
$$

where $C_{b}(e)$ is the betweenness of an edge which is defined as the sum of power transmitted pass through it between generation buses and load buses. 
$C_{b}(e)$ is expressed as follows:

$$
C_{b}(e)=\frac{\sum_{s \neq t} P_{s t}(e) / \sigma_{s t}}{\frac{n(n-1)}{2} P_{\sum l o a d}}
$$

where $P_{s t}(e)$ is the total power from the mentioned paths that conducts pass through branch $e$.

\subsection{Object function for full network observability}

The Optimal PMU Placement (OPP) problem is to determine the minimum number of PMUs and their corresponding locations in order to achieve full network observability. In other words, it needs to find one optimal solution for placing PMUs with minimum cost. The objective function to minimize the cost of PMUs is defined as follow:

$$
\begin{array}{ll} 
& \min ^{\sum_{k=1}^{n}} w_{k} x_{k} \\
\text { Subject to } & f(x)=A_{w} x>0
\end{array}
$$

where $x_{k}$ is a binary variable which is 0 or 1 whether PMU has been placed at bus or not; $w_{k}$ is the cost of the PMU installed at that bus; $A_{\mathrm{w}}$ is the weighted adjacency matrix of power system; $f(x)$ is a vector function, whose entries are non-zero if the corresponding bus voltage is solvable using the given measurement.

References [1-11, 24-26] accounted PMUs at every bus are the same cost, so the objective function of the minimum cost of PMUs corresponds to the minimum number of PMUs. However given the fact that, the cost corresponding to different PMUs are different due to the number of channels of each bus is not the same. Gholiha [13] provided the reference cost of each PMU according to the number of channels as shown in Table 1. Miljanić et al. [27] shown that it is not necessary to install PMU branch measurements in all branches incident to the bus where PMU is located. Therefore, in above-mentioned studies, the minimum number of PMUs do not correspond to the minimum total cost.

In this study, OPP is not only to specify the minimum number of PMUs but also to determine the number of channel of each PMU and their placement in the grid network. In addition, this study also considers the aspect of

Table 1. The cost of each PMU with respect to each channel

\begin{tabular}{c|c}
\hline Number of channels & Cost $(\$)$ \\
\hline 1 & 1500 \\
\hline 2 & 2000 \\
\hline 3 & 2500 \\
\hline 4 & 3000 \\
\hline 5 & 3500 \\
\hline 6 & 4000 \\
\hline 7 & 4500 \\
\hline 8 & 5000 \\
\hline
\end{tabular}

disposing reasonably of the number of channel of PMUs.

To assess the effectiveness of one OPP solution, with the exception of total cost we provide one index, which is named observation reliability of bus (BOR). This index is known as the observation reliability of bus. It depends on the reliability of PMU and line as well as the number of redundancies, and given by:

$$
\rho_{b}=1-\prod_{i=1}^{m}\left(1-\rho_{l i} \cdot \rho_{P M U i}\right)
$$

where $\rho_{b}$ is the observation reliability of bus; $\rho_{l}$ is the reliability of line which connects from PMU bus to this measurement bus; $\rho_{P M U}$ is the reliability of PMU; and $m$ is number of redundancies for this measurement bus.

Furthermore, in the case of the power grid network containing the zero injection buses, it is noted that the necessary number of PMUs may be reduced if some processing method is applied. Topology transformation and nonlinear constraint functions methods were proposed to take into account the zero injection buses [2, 4, 10, 11]. In this paper, we select the topology transformation for considering this problem.

\subsection{Observability redundancy}

The measurement redundancy is applied to enhance the reliability of system monitoring when considering the outage of PMU. In previous studies, it is passive because it happens casually when solving the observability problem. In this study, the measurement redundancy is selected actively. The criterion of selection which bus needs to put the measurement redundancy is its ODI value. The constraint vector function is modified as:

$$
\left\{\begin{array}{c}
f_{i}>0 \\
n z\left(f_{i}\right)=m
\end{array}\right.
$$

where $f_{i}$ is constraint function of bus i; $n z$ is the number of non-zero elements in $f_{i}$ function, and $m$ is a number of measurement redundancies for bus $i$.

To assess the effectiveness of redundancy, we compare the sum of ODI values of buses which have measurement redundancy.

\subsection{Observability in contingency modes}

The power system may face many kinds of contingencies in operation process. Among them, PMU failure and line outage are two most popular events that may affect on the system observability and the system monitoring. These conditions need to be considered when solving the OPP, and redundancy is one useful solution to overcome the unobservable situation.

Observability security index (OSI) is normally used to assess the effectiveness of OPP solution in contingency mode. OSI is given as follows: 


$$
O S I=\frac{r}{n}
$$

where $r$ is equivalent to the number of buses which can be observed when one PMU failure; and $n$ is the number of buses in the system.

\section{PMU Placement Algorithm}

In the proposed algorithm, the importance of buses and the reliability of branches are firstly calculated to form the weighted adjacent matrix. Subsequently, the prim's algorithm (PA) and the local search algorithm (LSA) are applied to find the optimal placement of PMUs in the power system. Based on the matrix $A_{w}$, the PA always can find the minimum structure of the graph, which ensures the number of branches with the highest reliability connected to all buses in power grid. Then LSA optimizes the number of PMUs. The PA fuses with LSA for optimizing both the number of branches of PMU and number of PMUs. It means that the electric power system is observed by a minimum number of PMUs with minimum channels.

Some following rules for PMU placement are assigned before starting the algorithm:

Rule 1: Assign one voltage measurement to a bus where a PMU has been placed.

Rule 2: If the voltage phase and current phase at one end of a branch are known, the voltage phase at the other end of the branch can be calculated by using line impedance.

Rule 3: If voltage phase at both ends of a branch is known, the branch current can be calculated by using line impedance.

The procedure for optimizing the placement of PMUs in power network based on the reliability of branches and the importance of buses is presented as follows:

Step 1. Finding the importance of buses and the reliability of branches:

The importance of buses is determined according to the calculating result of Eq. (2) and Eq. (4) is used to evaluate the reliability of branches. An algorithm is derived by Flament [28] to be applied to calculate $\sigma_{s t}$.

Step 2. Forming the weighted adjacent matrix.

Step 3. Defining the redundancy requirement for the measurement system.

Step 4. Finding the minimum connectivity structure of power grid network by PA:

PA is carried out by choosing an initial edge of minimum weight and successively adding edges of maximum weight that is incident to a node in the tree and that do not form simple circuits. Sequence of steps in executing the PA as follows:

(1) Input the weighted adjacent matrix $\left(A_{w}\right)$.

(2) Initialize a tree with a single node.

(3) Grow the tree by one edge: Find out the edge that is incident to the above-selected node, which has the largest reliability. Go through it whether forms a simple circuit, if not transfer it to the tree.

(4) Repeat the growing of tree until all nodes are in the tree.

After finishing the searching process by PA we obtain a weighted connectivity matrix (UG) which describes the minimum connectivity structure of power grid network with links having high reliability. In the executing process of PA, if there is more than one edge having the same weight that satisfies the appropriate criteria, the deterministic choice will rely on the importance of a bus.

Step 5. Finding the optimal placement of PMUs:

Here, local search algorithm has been chosen for finding out the optimal placement of PMUs. The idea of the method is each time to start from a different initial configuration. Searching progress is started from a set of buses having maximum importance. Procedure for finding out optimal location of PMU as follows:

(1) Finding out the radial buses in the network (radial bus is the bus which connected to only one other bus) from matrix UG.

(2) Forming a set of buses that having maximum importance; then, checking whether any buses in this set connected to a radial bus that will be chosen to place PMU. If the set of the radial bus is null then the bus having highest importance will be PMU bus.

(3) Finding directly observable buses and total observable buses (TOB) after placing PMU at bus selected. Check TOB if it is equal to the total number of bus of system then system will be completely observable, continue moving to Step 6, otherwise reform the matrix UG then go back to the finding process.

Step 6. Supplement the measurement redundancies:

From the list of buses with redundancy defined in Step 3, supplementing the measurement redundancy for those buses from PMU which placed at adjacent bus if having, or if not then adding a new PMU for obtaining the maximum redundancy.

Step 7. Reduce the duplicated channels to optimize the total cost:

Find the non-PMU buses which are connected to more than one PMU bus (except the redundant buses). Select the branch which has larger reliability. If there are more branches with the same value then the branch connected to PMU bus, which has less number of channels, will be chosen.

Considering a small 7 bus test system as shown in Fig. 1;

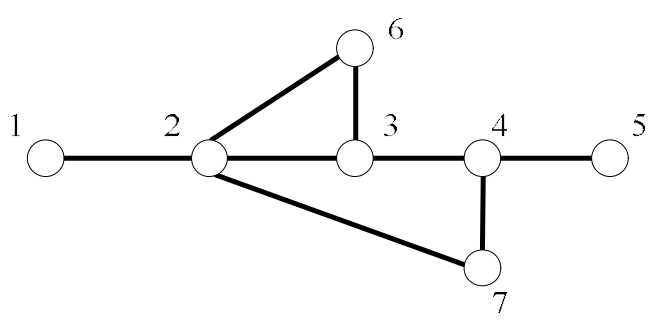

Fig. 1. Seven bus test system 
Table 2. Power distribution on 7 bus system

\begin{tabular}{cccccccc}
\hline Bus & 1 & 2 & 3 & 4 & 5 & 6 & 7 \\
\hline Power $(\mathrm{MW})$ & Generation & 20 & 15 & 30 & 5 & 5 & 10 \\
\hline
\end{tabular}

Table 3. Line parameters of 7 bus system

\begin{tabular}{lcccc}
\hline & From & To & $\mathbf{X}(\boldsymbol{\Omega})$ & $\boldsymbol{\lambda}$ \\
\cline { 2 - 5 } Line 1 & 1 & 2 & 0.05 & 0.02 \\
Line 2 & 2 & 3 & 0.1 & 0.04 \\
Line 3 & 2 & 6 & 0.2 & 0.16 \\
Line 4 & 2 & 7 & 0.5 & 0.29 \\
Line 5 & 3 & 4 & 0.1 & 0.05 \\
Line 6 & 3 & 6 & 0.15 & 0.11 \\
Line 7 & 4 & 5 & 0.25 & 0.22 \\
Line 8 & 4 & 7 & 0.2 & 0.16 \\
\hline
\end{tabular}

it also supposes that the information about power value of each bus, reactance $(\mathrm{X})$ and outage rate $(\lambda)$ of each line is known as in Table 2 and Table 30

In step 1, the ODI of buses in the 7 bus test system specified as follows:

$\begin{array}{lccccccc}\text { Bus } & 1 & 2 & 3 & 4 & 5 & 6 & 7 \\ \text { ODI } & 1.00 & 1.00 & 0.71 & 0.53 & 0.06 & 0.06 & 0.12\end{array}$

and the reliability of each line:

$\begin{array}{ccccccccc}\text { Line } & 1 & 2 & 3 & 4 & 5 & 6 & 7 & 8 \\ \rho & 0.93 & 0.93 & 0.85 & 0.75 & 0.93 & 0.90 & 0.80 & 0.85\end{array}$

Move to step 2, the $A_{w}$ matrix is formed as follows:

$$
\left[\begin{array}{ccccccc}
1 & 0.93 & 0 & 0 & 0 & 0 & 0 \\
0.93 & 1 & 0.93 & 0 & 0 & 0.85 & 0.75 \\
0 & 0.93 & 0.71 & 0.93 & 0 & 0.9 & 0 \\
0 & 0 & 0.93 & 0.53 & 0.8 & 0 & 0.85 \\
0 & 0 & 0 & 0.8 & 0.06 & 0 & 0 \\
0 & 0.85 & 0.9 & 0 & 0 & 0.06 & 0 \\
0 & 0.75 & 0 & 0.85 & 0 & 0 & 0.12
\end{array}\right]
$$

After applying the PA to select the most reliable connections in step 4 we obtain the UG matrix as below:

$$
\left[\begin{array}{ccccccc}
1 & 0.93 & 0 & 0 & 0 & 0 & 0 \\
0.93 & 1 & 0.93 & 0 & 0 & 0 & 0 \\
0 & 0.93 & 0.71 & 0.93 & 0 & 0.9 & 0 \\
0 & 0 & 0.93 & 0.53 & 0.8 & 0 & 0.85 \\
0 & 0 & 0 & 0.8 & 0.06 & 0 & 0 \\
0 & 0 & 0.9 & 0 & 0 & 0.06 & 0 \\
0 & 0 & 0 & 0.85 & 0 & 0 & 0.12
\end{array}\right]
$$

From the UG matrix, we first check the number of nonzero elements in each row (equate to 2) to find out the set of radial buses. This set consists of four buses 1, 5, 6 and 7 . Subsequently, forming the PMU set: bus 2 having the highest ODI value so it will be checked first. Since bus 2 connects to bus 1 so it is added into PMU set; bus 3 continues to be checked and then move to bus 4 . Both these buses are all added into PMU set because they connect directly to radial buses; bus 3 connects to bus 6 , then bus 4 has the connection with bus 5 and bus 7. After the first iteration, the obtained PMU set consists of three buses 2,3 and 4 that can cover fully the network $(\mathrm{TOB}=7)$ so the searching progress finishes and moves to step 6 .

In step 6, the redundancy is considered for high important buses. Bus 1 and bus 2 are the most important; bus 3 and bus 4 also have high importance. However, bus 1 is radial bus; therefore, the redundancy is considered for only three buses 2, 3 and 4. With the obtained PMU set in step 5, all these buses have the measurement redundancy so the process continues moving to step 7 to reduce the duplicated measurement channels. In the obtained PMU

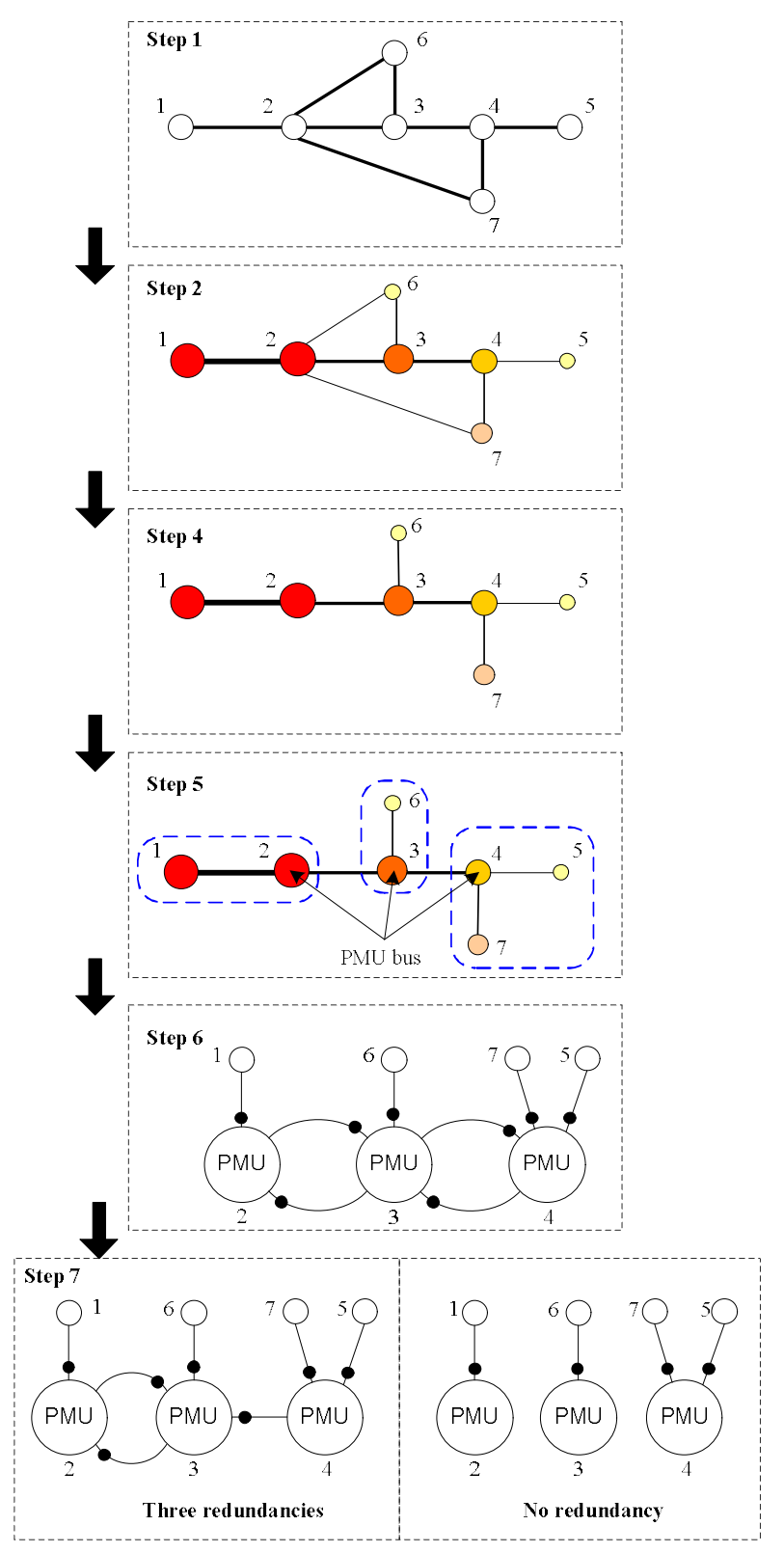

Fig. 2. Flow of PMU placement on graph for 7 bus system 
placement, there is only one duplicated channel needed to remove, that is 4-3; then the final placement of PMU is (2$1,2-3 ; 3-2,3-4,3-6 ; 4-5,4-7)$. In the case of having no redundant requirement, the channels $2-3,3-2$ and $3-4$ will be removed; the final placement is $(2-1 ; 3-6 ; 4-5,4-7)$.

The detail result of each step on the graph is illustrated in Fig. 2.

\section{Simulations and Results}

Simulations on the IEEE 14, IEEE 24, IEEE 30 and IEEE 57 bus systems are carried out in MATLAB. The information of the four systems is available in [29]. The simulation process has been done in three stages:

Stage 1: Programming to calculate the reliability of lines and the importance of buses.

Stage 2: Testing and justifying the proposed algorithm on IEEE test systems.

Stage 3: Calculating the indices, which is used to assess and compare the effects of this study with other previous studies.

Fig. 3 shows the observation reliability of buses in the test systems. In simulation situation, the outage rate of lines $(\lambda)$ in Eq.(4) is calculated according to power and voltage grade under the assumption of an equal length of $100 \mathrm{~km}$ for all the network lines. The zero-injection buses had been reduced so their values are zero (bus 7 in 14-bus system); and with supposing that the reliability of PMU is one, then the observation reliability of buses which PMU placed are also one (buses 4, 5, 6, 8, 9 and 13 in 14-bus system). The observation reliability of bus consequently depends on the reliability of branch and number of redundancies.

The observation degree index of buses is shown in Fig. 4.
Table 5. Number and location of PMUs with full network observation

\begin{tabular}{|c|c|c|c|c|c|c|}
\hline \multirow{2}{*}{ System } & \multirow{2}{*}{$\begin{array}{l}\text { Number } \\
\text { of PMUs }\end{array}$} & \multicolumn{4}{|c|}{ Location of PMUs } & \multirow{2}{*}{$\begin{array}{c}\text { Cost } \\
(\$)\end{array}$} \\
\hline & & Bus & & Channel & & \\
\hline \multirow{5}{*}{ 14-bus } & \multirow{5}{*}{5} & 4 & $4-3$ & $4-7$ & - & \multirow{5}{*}{9500} \\
\hline & & 5 & $5-1$ & $5-2$ & - & \\
\hline & & 6 & $6-11$ & - & - & \\
\hline & & 9 & $9-10$ & - & - & \\
\hline & & 13 & $13-12$ & $13-14$ & - & \\
\hline \multirow{8}{*}{ 24-bus } & \multirow{8}{*}{8} & 1 & $1-2$ & $1-5$ & - & \multirow{8}{*}{15000} \\
\hline & & 8 & $8-7$ & - & - & \\
\hline & & 9 & $9-4$ & $9-11$ & $9-12$ & \\
\hline & & 10 & $10-6$ & - & - & \\
\hline & & 15 & $15-13$ & - & - & \\
\hline & & 16 & $16-14$ & $16-19$ & - & \\
\hline & & 21 & $21-18$ & $21-22$ & - & \\
\hline & & 23 & $23-13$ & $23-20$ & - & \\
\hline \multirow{9}{*}{ 30-bus } & \multirow{9}{*}{9} & 3 & $3-1$ & - & - & \multirow{9}{*}{17500} \\
\hline & & 4 & $4-2$ & - & - & \\
\hline & & 5 & $5-7$ & - & - & \\
\hline & & 6 & $6-8$ & $6-9$ & - & \\
\hline & & 10 & $10-17$ & $10-20$ & $10-21$ & \\
\hline & & 12 & $12-13$ & $12-14$ & $12-16$ & \\
\hline & & 18 & $18-15$ & $18-19$ & - & \\
\hline & & 24 & $24-23$ & $24-25$ & - & \\
\hline & & 27 & $27-29$ & $27-30$ & - & \\
\hline \multirow{14}{*}{ 57-bus } & \multirow{14}{*}{14} & 1 & $1-2$ & $1-16$ & $1-17$ & \multirow{14}{*}{29500} \\
\hline & & 6 & $6-4$ & $6-5$ & - & \\
\hline & & 9 & $9-12$ & $9-13$ & - & \\
\hline & & 15 & $15-3$ & $15-44$ & - & \\
\hline & & 19 & $19-18$ & $19-20$ & - & \\
\hline & & 25 & $25-23$ & $25-27$ & $25-30$ & \\
\hline & & 29 & $29-7$ & $29-28$ & $29-52$ & \\
\hline & & 32 & $32-31$ & $32-33$ & - & \\
\hline & & 37 & $37-36$ & $37-38$ & - & \\
\hline & & 43 & $43-11$ & $43-41$ & - & \\
\hline & & 47 & $47-14$ & $47-49$ & - & \\
\hline & & 51 & $51-10$ & $51-50$ & - & \\
\hline & & 54 & $54-53$ & $54-55$ & - & \\
\hline & & 56 & $56-42$ & $56-57$ & - & \\
\hline
\end{tabular}

Table 4. Position of branches with high power density and buses with high importance in IEEE 14, 24, 30 and 57 bus systems

\begin{tabular}{r|c|c|c|c}
\hline System & 14-bus & 24-bus & 30 -bus & 57-bus \\
\hline $\begin{array}{c}\text { Branches with high power } \\
\text { density }\end{array}$ & $\begin{array}{c}1-2,2-4,2-3,4-9, \\
1-5,5-6\end{array}$ & $14-16,11-14,15-16,10-11$, & $1-2,2-5,2-6,6-10,4-12$, & $11-13,9-11,12-13,13-15,3-15,8-9$, \\
$6-28,12-15$ & $3-4,14-47,1-15,4-6$ \\
\hline Buses with high importance & $2,4,9,5,6$ & $16,10,11,14,15,5,19,8,23$ & $6,2,4,10,12,27,15$ & $12,13,15,11,1,14,3,4,8,9,47$, \\
\hline
\end{tabular}
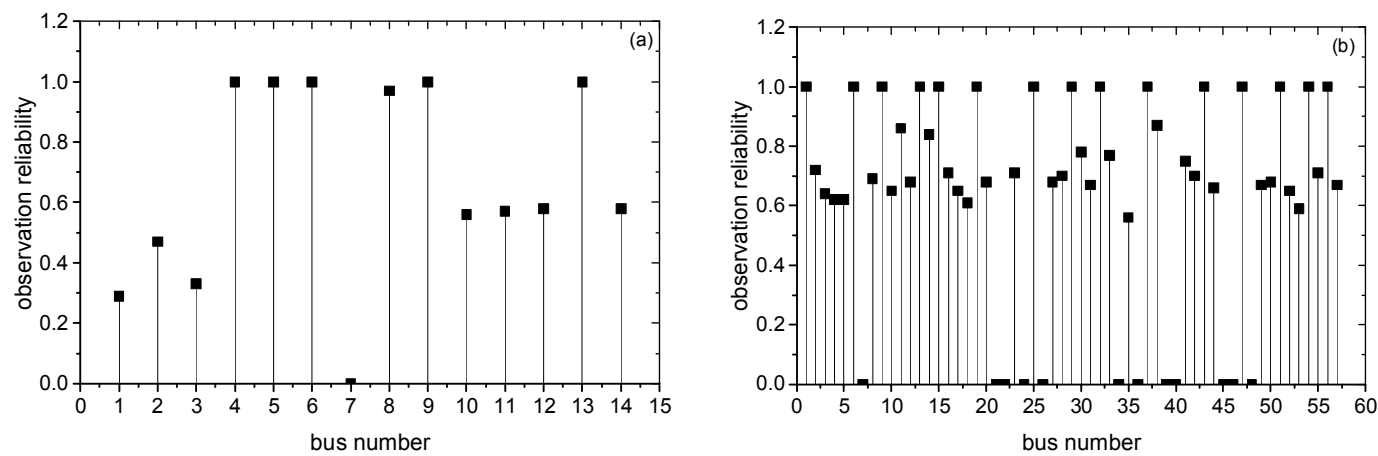

Fig. 3. Observation reliability of bus: (a) 14 bus system; (b) 57 bus system 
This figure points out that the buses 2, 4, 9 in the 14-bus system and the buses $12,13,15,11$ in the 57-bus system are the most important buses. They are electrical hubs of the power network.

Table 4 lists a number of buses and branches which having high importance and high power density in all test systems. These are the buses having the highest ODI value, so they have a high priory in considering the measurement redundancy. The branches with high power conducted on are the most vulnerable lines, then having a low priory in selecting to place PMU.

The bus number and branches of PMUs obtained from proposed method as well as total cost of PMUs arepresented in Table 5 considering only the observation, and in Table 6 for the selected buses measurement redundancy to be added. In two tables, the column $2^{\text {nd }}$ expresses the number of PMUs and the column $3^{\text {rd }}$ shows the location of PMUs and their current measurement channels. The selected branches of PMUs all have a lower rate of outage than other selections; for example, bus 1 in the 14 bus system can be observed from bus 2 or bus 5 through line 1-5 or line 1-2. Line 1-5 has a high outage rate, but it is still lower than line 1-2; hence, line 1-5 is a better selection. We also see that almost high important buses were placed PMU (the buses 4, 9, 5, 6 in 14 bus system; the buses $10,16,15,8,23$ in 24 bus system; the buses 4,6 , 10, 12, 27 in 30 bus system; the buses 15, 9, 1, 47, 29, 37 in 57 bus system); however, some the most important buses were not selected to install PMU (for example, bus 1 and bus 2 in 14 bus system) because they are radial buses in the UG matrix, or they do not have directed connection with any radial buses so that they were not checked first, and then the lower important buses were selected. The column $4^{\text {th }}$ of Table 6 presents the selected buses with the measurement redundancy. In this study, the number of redundant buses should be selected to compare fairly with other methods. With the 14-bus test system, the selected important buses are the buses 2, 4, 5, 6 and 9; the 24-bus

Table 6. Number and location of PMUs with considering the measurement redundancy

\begin{tabular}{|c|c|c|c|c|c|c|c|c|}
\hline \multirow{2}{*}{ System } & \multirow{2}{*}{ Number of PMUs } & \multicolumn{5}{|c|}{ Location of PMUs } & \multirow{2}{*}{$\begin{array}{l}\text { Buses with measurement } \\
\text { redundancy }\end{array}$} & \multirow{2}{*}{$\begin{array}{l}\text { Cost } \\
(\$)\end{array}$} \\
\hline & & Bus & \multicolumn{4}{|c|}{ Channels } & & \\
\hline \multirow{5}{*}{ 14-bus } & \multirow{5}{*}{5} & 4 & $4-3$ & $4-7$ & $4-9$ & $4-2$ & \multirow{5}{*}{$2,4,5,6,9$} & \multirow{5}{*}{11500} \\
\hline & & 5 & $5-1$ & $5-2$ & $5-4$ & - & & \\
\hline & & 6 & $5-11$ & $6-5$ & - & - & & \\
\hline & & 9 & $9-10$ & - & - & - & & \\
\hline & & 13 & $13-12$ & $13-14$ & $13-6$ & - & & \\
\hline \multirow{9}{*}{ 24-bus } & \multirow{9}{*}{9} & 1 & $1-2$ & $1-5$ & - & - & \multirow{9}{*}{$16,10,11,14,15,5,8$} & \multirow{9}{*}{19000} \\
\hline & & 8 & $8-7$ & $8-10$ & - & - & & \\
\hline & & 9 & $9-4$ & $9-11$ & $9-8$ & - & & \\
\hline & & 10 & $10-6$ & $10-12$ & $10-5$ & - & & \\
\hline & & 11 & $11-4$ & - & - & - & & \\
\hline & & 15 & $15-13$ & $15-16$ & - & - & & \\
\hline & & 16 & $16-14$ & $16-19$ & - & - & & \\
\hline & & 21 & $21-18$ & $21-22$ & $21-15$ & - & & \\
\hline & & 23 & $23-13$ & $23-20$ & - & - & & \\
\hline \multirow{9}{*}{ 30-bus } & \multirow{9}{*}{9} & 3 & $3-1$ & $3-4$ & - & - & \multirow{9}{*}{$6,2,4,10,12,27,15$} & \multirow{9}{*}{21000} \\
\hline & & 4 & $4-2$ & $4-6$ & $4-12$ & - & & \\
\hline & & 5 & $5-7$ & $5-2$ & - & - & & \\
\hline & & 6 & $6-8$ & $6-9$ & $6-28$ & - & & \\
\hline & & 10 & $10-17$ & $10-20$ & $10-21$ & - & & \\
\hline & & 12 & $12-13$ & $12-14$ & $12-15$ & $12-16$ & & \\
\hline & & 18 & $18-15$ & $18-19$ & - & - & & \\
\hline & & 24 & $24-23$ & $24-22$ & $24-25$ & - & & \\
\hline & & 27 & $27-29$ & $27-30$ & - & - & & \\
\hline \multirow{15}{*}{ 57-bus } & \multirow{15}{*}{15} & 1 & $1-2$ & $1-15$ & $1-16$ & $1-17$ & \multirow{15}{*}{$12,13,15,11,14,38$} & \multirow{15}{*}{33000} \\
\hline & & 6 & $6-4$ & $6-5$ & - & - & & \\
\hline & & 9 & $9-12$ & $9-13$ & - & - & & \\
\hline & & 13 & $13-11$ & $13-12$ & $13-14$ & - & & \\
\hline & & 15 & $15-3$ & $15-44$ & - & - & & \\
\hline & & 19 & $19-18$ & $19-20$ & - & - & & \\
\hline & & 25 & $25-23$ & $25-27$ & $25-30$ & - & & \\
\hline & & 29 & $29-7$ & 29-28, & $29-52$ & - & & \\
\hline & & 32 & $32-31$ & $32-33$ & - & - & & \\
\hline & & 37 & $37-36$ & $37-38$ & - & - & & \\
\hline & & 43 & $43-11$ & $43-41$ & - & - & & \\
\hline & & 47 & $47-14$ & $47-49$ & $47-38$ & - & & \\
\hline & & 51 & $51-10$ & $51-50$ & - & - & & \\
\hline & & 54 & $54-53$ & $54-55$ & - & - & & \\
\hline & & 56 & $56-42$ & $56-57$ & - & - & & \\
\hline
\end{tabular}



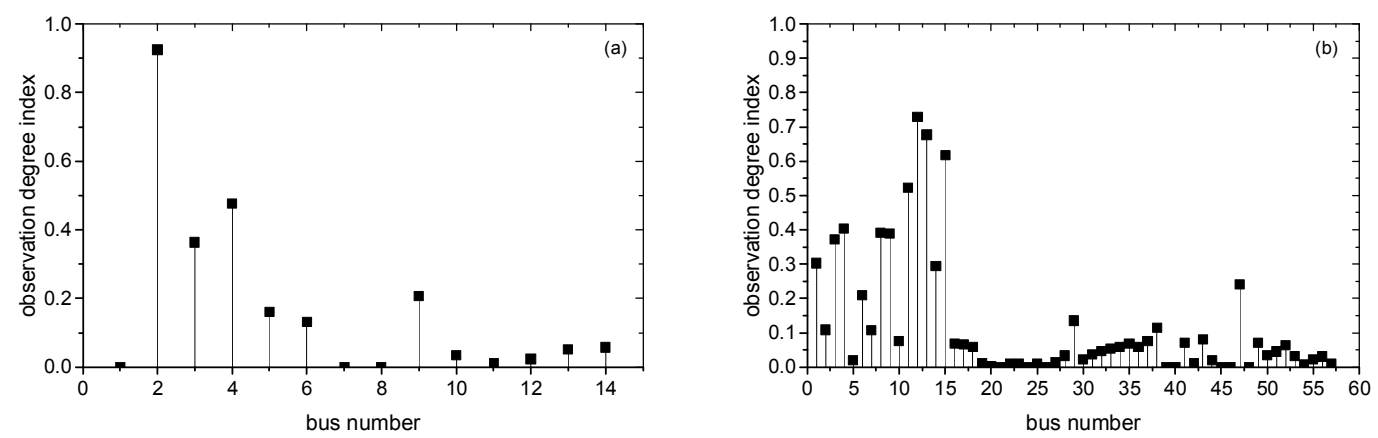

Fig. 4. Observation degree index of bus: (a) 14 bus system; (b) 57 bus system
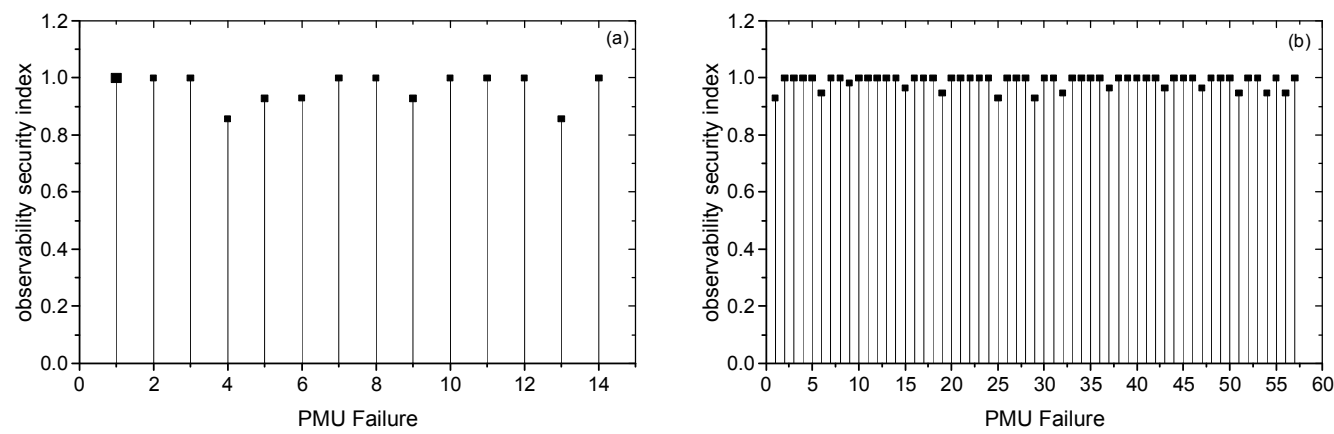

Fig. 5. Observability security index: (a) 14 bus system; (b) 57 bus system

Table 7. Simulation results of the IEEE 30 bus system in some previous studies

\begin{tabular}{c|c|c|c|c}
\hline References & Number of PMUs & Places of PMUs & $\begin{array}{c}\text { Buses with measurement } \\
\text { redundancy }\end{array}$ & $\begin{array}{c}\text { Cost } \\
(\$)\end{array}$ \\
\hline$[11]$ & 7 & $2,4,10,12,15,20,27$ & $2,4,6,10,12,15,20$ & 21500 \\
\hline$[6]$ & 7 & $2,3,10,12,15,19,27$ & $1,4,6,12,15,18,20$ & 20500 \\
\hline$[24]$ & 7 & $2,4,10,12,15,19,27$ & $2,4,6,12,15,18,20$ & 21500 \\
\hline$[13]$ & 15 & $2-1,4-3,5-7,6-8,9-11,10-20,12-13,14-15,16-17,18-19$, & - & 22500 \\
\hline
\end{tabular}

Table 8. Simulation results of the IEEE 57 bus system in some previous studies

\begin{tabular}{c|c|c|c|c}
\hline References & Number of PMUs & Places of PMUs & Buses with measurement redundancy & Cost (\$) \\
\hline$[11]$ & 11 & $1,4,13,20,25,29,32,38,51,54,56$ & 15,49 & 32000 \\
\hline$[6]$ & 12 & $1,6,9,15,20,25,29,32,38,51,53,56$ & $1,8,10,13,15$ & 35500 \\
\hline$[24]$ & 11 & $1,5,13,19,25,29,32,38,41,51,54$ & 15,49 & 31000 \\
\hline
\end{tabular}

system includes bus 5 , bus 8 , bus 10 , bus 11 , bus 14 , bus 15 and bus 16; then, the buses 2, 4, 6, 10, 12, 15 and 27 were chosen for 30-bus system. In the 57-bus system, the buses $12,13,15,11,14$, and 38 are the most important buses, so they were chosen. The last column calculates the total cost of PMUs. From these tables, we can see that the cost increases according to the number of redundancies (500\$ per one redundant channel).

After the process of PMU placement finished, the observability of power system was assessed in the contingency mode with the failure of one PMU. Fig. 5 illustrates the observability security index of the 14 and 57 bus test systems based on the obtained PMU placement. The value of OSI increases from zero to one; the OSI value of non-PMU buses are one. In proposed method, the optimization reduces the number of PMU channels, therefore, the value of OSI increases. As shown in this figure, all values of OSI are high. The average value of OSI is 0.91 with the 14 -bus system and is 0.95 with the 57 bus system.

Table 7 presents the results of simulation on the IEEE 30 -bus system that were analyzed in $[6,11,13,24]$. All methods ensure complete system observability with the total cost is approximately 21500 (\$). Except [13] has no redundancy, refs. [6, 11, 24] have seven redundant buses; however, buses 18 and 20 are not important. The proposed method also have seven redundancies; these buses are all important. Moreover, the installation cost of the proposed method is only 21000 (\$). Similarly, Table 8 shows the results of simulation on IEEE 57 bus system. In $[11,24]$, the optimal number of PMUs is 11 that still ensures full network observation; the total cost is 31000 (\$), but the 
number of redundancies is only 2. In [6], the number of redundancies is 5 ; however, the cost increases to 35500 (\$). Comparing with the proposed approach, its cost is 33000 (\$) and it has six important redundant buses. As we can see, although the number of PMUs in proposed method is larger than that in previous methods, the total cost is still remained or even less than it in previous methods. Moreover, the redundancy is all for important buses. In addition, from Table 7 and Table 8 can see clearly that the redundancy of previous studies is passive: In the 30-bus system, the number of redundancies is 7 , but it reduces to 2 in the 57-bus system. On the contrary, this problem is completely active in the proposed method; the redundancy can implement for any specific buses.

Table 9 shows the average values of OSI for IEEE 14, 30 and 57 bus system. In this table, these results also compare with four other references. As seen with the 14-bus system, the OSI values of $[6,11,13,24]$ are only 0.71 and 0.86 while it is 0.91 for proposed method; with the 30 bus and 57 bus systems, they are 0.93 and 0.95 . Although the main purpose of [13] is to maximize the values of OSI, its value is not much higher than the value of proposed method; it is even lower in the case of the IEEE 14 bus system. From this table, it can be said that the security of observability, which is achieved by the proposed method, is much better than other methods except reference [13]. This can be obtained because the proposed algorithm applied one solution which reduces the number of channels and increases the number of PMUs to keep the total cost.

Average values of BOR for IEEE 14, 30 and 57 bus systems are shown in Table 10. This table demonstrates the salient effect of the proposed method because all values of the BOR, which calculated by this method, are larger than that of other methods. In $[6,11,13,24]$, the average values of BOR are $0.64,0.57$ and 0.75 for IEEE 14, 30 and 57 bus systems, respectively; while those are $0.70,0.64$ and 0.81 for the proposed method. The proposed approach applied the Prim algorithm to specify the most reliable channel for

Table 9. Average values of OSI for IEEE 14, 30 and 57 bus systems

\begin{tabular}{c|c|c|c}
\hline System & 14-bus & 30-bus & 57-bus \\
\hline$[11]$ & - & 0.89 & 0.93 \\
\hline$[6]$ & 0.71 & 0.88 & 0.93 \\
\hline$[24]$ & 0.71 & 0.89 & 0.92 \\
\hline$[13]$ & 0.86 & 0.93 & 0.96 \\
\hline Proposed method & 0.91 & 0.93 & 0.95 \\
\hline
\end{tabular}

Table 10. Average values of BOR for IEEE 14, 30 and 57 bus systems

\begin{tabular}{c|c|c|c}
\hline System & 14-bus & 30-bus & 57-bus \\
\hline$[11]$ & 0.64 & 0.56 & 0.75 \\
\hline$[6]$ & 0.64 & 0.60 & 0.76 \\
\hline$[24]$ & 0.64 & 0.57 & 0.75 \\
\hline Proposed method & 0.70 & 0.64 & 0.81 \\
\hline
\end{tabular}

each bus; hence, it enhances not only the observation reliability of each bus but also the observation reliability of system.

The results of the simulations in the test systems provide more insight into the power network observation reliability and the optimal PMU placement problem. Based on the findings of this study, one valuable foundation can develop to perfect the optimal PMU placement method for a given power network.

\section{Discussions}

This study presents an approach for PMU placement based on a modified weighted adjacent matrix that concerns about not only the size, the connectivity characteristics but also the electrical characteristics of power network (generation location, load center, power transferred station). The following salient features should be considered in this algorithm:

- PMUs are placed at the important buses that both ensures the observability and enhances the effect in operating, monitoring and assessing for the power system.

- The bus observation reliability is calculated based on not only the reliability of PMU and the redundancy but also the reliability of transmission lines that will maximize the observation reliability for buses.

- Reducing the number of channels and increasing the number of PMUs to keep the total cost, this is not only improving the OSI but also extending the redundancy.

- The active redundancy helps enhance the observable effect and the system operation ability in the contingency mode.

- The PMU placement determines specifically the channels of each PMU that prevent the case of duplicated measurement.

- The number of measurement channels at each PMU station is also considered to allocate in the balance; it can help overcome the lack of communication facilities and the limit of storage as well as transmitted rate.

- Because the importance of buses and the reliability of channels relate to the location as well as the value of loads and generations so the change of major load center or/and major generation center will have a certain impact on the effect of the proposed method. In that case, the bus observation reliability may be changed; however, the effective observability is not affected.

\section{Conclusions}

This paper proposed a new method to obtain full network observability as well as the measurement redundancy through solving the optimal PMU placement problem. The proposed method is based on the reliability 
of branches and the importance of buses to optimize both PMUs and their channels. It can improve significantly the observation reliability of the system with the selection of high reliable branches, the redundancy for important buses and the high OSI. The effect of this method was demonstrated by simulating on the IEEE 14, 24, 30 and 57 bus test systems. The results of this study showed that the proposed method has many advantages compared to previous methods, such as ensuring the effective observability, low cost and maximizing the observation reliability of the system.

\section{Acknowledgment}

The authors of this paper would like to acknowledge the support of the Science and technology Development Plan of China Railway (Grant 2015Z005-D) and the National Natural Science Foundation of China (Grant 61174179).

\section{References}

[1] Gou, B., "Optimal Placement of PMUs by Integer Linear Programming," IEEE Transaction on Power Systems, vol. 23, no. 3, pp. 1525-1526, 2008.

[2] $\mathrm{Xu}, \mathrm{B}$. and A. Abur, "Observability Analysis and Measurement Placement for Systems with PMUs," in Power Systems Conference \& Exposition, pp. 943946, 2004.

[3] Naydu, G.V. and A.B. Parmar, "Optimal Placement of Phasor Measurement Units using Integer Linear Programming," International Journal of Engineering Development and Research, vol. 2, no. 2, pp. 25442549, 2014.

[4] Dua, D., S. Dambhare, and R.K. Gajbhiye, "Optimal Multistage Scheduling of PMU Placement: An ILP approach," IEEE Transactions on Power Delivery, vol. 23, no. 4, pp. 1812-1820, 2008.

[5] P.Theodorakatos, N., N.M. Manousakis, and G.N. Korres, "Optimal Placement of Phasor Measurement Units with Linear and Non-linear Models," Electric Power Components and Systems, vol. 43, no. 4, pp. 357-371, 2015.

[6] Shewale, R.H., et al., "Optimal Placement of Phasor Measurement Unit for Power System Observability by Heuristic Search Method," International journal of Advanced Technology \& Engineering Research, vol. 2, no. 2, pp. 128-133, 2012.

[7] Peng, J., Y. Sun, and H.F. Wang, "Optimal PMU Placement for Full Network Observability using Tabu Search Algorithm," Electrical Power and Energy Systems, vol. 28, pp. 223-231, 2006.

[8] Hurtgen, M. and J.-C. Maun, "Optimal PMU Placement using Iterated Local Search," Electrical Power and Energy Systems, vol. 32, pp. 857-860, 2010.
[9] Puri, K. and D.G.S. Brar, "Optimal Placement of Phasor Measurement Units for Power System Obserbability Using A Hybrid of Genetic and Cuckoosearch (CS-GA) Algorithms," International Journal of Science and Research, vol. 4, no. 6, pp. 1006-1013, 2015.

[10] Ahmadi, A., Y. Alinejad-Beromi, and M. Moradi, "Optimal PMU Placement for Power System Observability using Binary Particle Swarm Optimization and Considering Measurement Redundancy," Expert Systems with Applications, vol. 38, pp. 72637269, 2011

[11] Mahaei, S. M. and M.T. Hagh, "Minimizing the Number of PMUs and Their Optimal Placement in Power Systems," Electric Power Systems Research, vol. 83, pp. 66-72, 2012.

[12] Dalali, M. and H.K. Karegar, "Optimal PMU Placement for Full Observability of the Power Network with Maximum Redundancy using Modified Binary Cuckoo Optimisation Algorithm," IET Generation, Transmission \& Distribution, vol. 10, no. 11, pp. 2817-2824, 2016.

[13] Gholiha, M.M. and R. Noorozian, "New Method Based on Branch for Optimal Placement of PMU using Harmony Search Algorithm," Majlesi Journal of Energy Management, vol. 3, no. 2, pp. 11-17, 2014

[14] Aghaei, J., et al., "Multi-objective Phasor Measurement Unit Placement in Electric Power Networks: Integer Linear Programming Formulation," Electric Power Components \& Systems, vol. 43, no. 17, pp. 1902-1911, 2015.

[15] Mousavian, S. and M.J. Feizollahi, "An Investment Decision Model for the Optimal Placement of Phasor Measurement Units," Expert Systems with Applications, vol. 42, no. 21, pp. 7276-7284, 2015.

[16] Abiri, E., F. Rashidi, and T. Niknam, "An Optimal PMU Placement Method for Power System Observability under Various Contingencies," International transactions on electrical energy systems, vol. $25, \mathrm{pp}$. 589-606, 2015.

[17] Vedik, B. and A.K. Chandel, "Optimal PMU Placement for Power System Observability using Taguchi Binary Bat Algorithm," Measurement, vol. 95, pp. 8-20, 2016.

[18] Xu, S., et al. "Vulnerability Assessment of Power Grid Based on Complex Network Theory," in Proceedings of the Asia-Pacific Power and Energy Engineering Conference (APPEEC'09), 2009.

[19] Freeman, L.C., "A set of Measures of Centrality Based on Betweenness," Sociametry, vol. 40, no. 1, pp. 35-41, 1977.

[20] Wang, Z., A. Scaglione, and R.J. Thomas, "Electrical Centrality Measures for Power Grids, in Control and Optimization Methods for Electric Smart Grids," M.D.I.c. A. Chakrabortty, Editor. 2012, Springer. pp. 239-255. 
[21] Gutierrez, F., et al., "Vulneriability Analysis of Power Grids Using Modified Centrality Measures," Discrete Dynamics in Nature and Society, pp. 1-11, 2013.

[22] Zio, E. and L.R. Golea, "Analyzing the Topological, Electrical and Reliability Characteristics of a Power Transmission System for Identifying its Critical Elements," Reliability Engineering and System Safety, vol. 101, pp. 67-74, 2012.

[23] Chen, X., et al., "Identification of Vulnerable Lines in Power Grid Based on Complex Network Theory," in International Conference on Mechatronic Science, Electric Engineering and Computer (MEC), 2011, pp. $118-121$.

[24] Mahari, A. and H. Seyedi, "Optimal PMU Placement for Power System Observability using BICA, Considering Measurement Redundancy," Electric Power Systems Research, vol. 103, pp. 78-85, 2013.

[25] Hajian, M., et al., "Optimal Placement of PMUs to Maintain Network Observability using a Modified BPSO Algorithm," Electrical Power and Energy Systems, vol. 33, pp. 28-34, 2011.

[26] Babu, R. and B. Bhattacharyya, "Optimal Allocation of Phasor Measurement Unit for Full Observability of the Connected Power Network," Electrical Power and Energy Systems, vol. 79, pp. 89-97, 2016.

[27] Miljanić, Z., I. Djurović, and I. Vujošević, “Optimal Placement of PMUs with Limited Number of Channels," Electric Power Systems Research, vol. 90, pp. 93-98, 2012.

[28] Flament and Claude, "Applications of Graph Theory to Group Structure," N.J. Englewood Cliffs Editor, Prentice Hall, 1963.

[29] http://www.ee.washington.edu/research/pstca. [cited 2016 May 12].

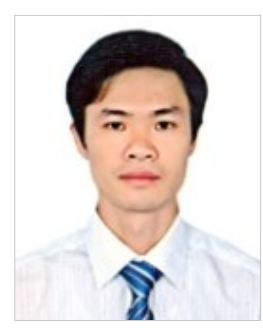

Van-Khoi TRAN He received B.S and M. S. degree in electric equipment and automation from the university of transport and communications, Hanoi, Vietnam in 2004 and 2009, respectively. $\mathrm{He}$ is currently a doctoral student at Beijing Jiaotong University, Beijing, China. His research interests are measurement technique and automation equipment.

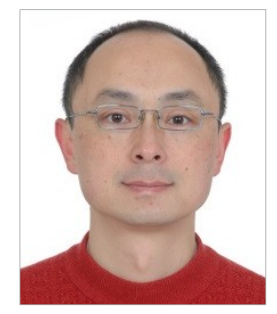

He-sheng ZHANG He received B.S and $\mathrm{M}$. S. degree in electrical engineering from Northern Jiaotong University (Beijing Jiaotong University) in 1992 and 1995, respectively, and received $\mathrm{Ph} . \mathrm{D}$ degree in department of automation from Tsinghua University in 2006. He is currently a professor and director of department of electric drive and control in Beijing Jiaotong Univeristy. His research interests are measurement technique and automation equipment.

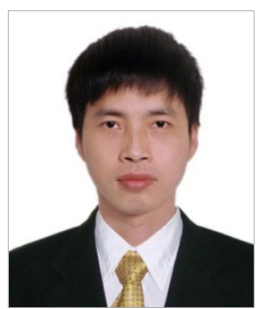

Van-Nghia NGUYEN He received B.S. degree from university of transport and communications, Hanoi, Vietnam(UTC) in 1995, M. S. degree in 2002, and received Ph.D. degree in Vietnam Institute of Electronics, Informatics and Automation in 2006. $\mathrm{He}$ is currently a vice director of department of electrical and Electronic Engineering in UTC. His research interests are power electronic, measurement technique and technical diagnosis. 